\title{
La adaptación a la tecnológica en la educación: una situación emergente
}

Dr.Isaí Alí Guevara Bazán, Mexicano.

ORCID: https://orcid.org/0000-0002-1500-291X

iguevara@uv.mx

Dr. Jorge Martínez Cortés, Mexicano.

ORCID: https://orcid.org/0000-0002-8696-2088

jomartinez@uv.mx

Mtro. Ángel Augusto Landa Alemán, Mexicano.

ORCID: https://orcid.org/0000-0002-1454-2236

anlanda@uv.mx

Universidad Veracruzana, México

Recibido: 24 de junio del 2020

Aceptado: 25 de agosto del 2020

\section{Resumen}

Tras la emergencia de la contingencia COVID, la impartición de clases presenciales fue imposible y la emigración a la modalidad virtual presentó retos para los docentes, los cuales tuvieron que adaptarse a los medios tecnológicos. Sin embargo, se prefiere la capacitación en el uso de las TIC para evitar inconsistencias al diseñar e implementar procesos innovadores. Esto nos lleva a la necesidad de los docentes de adaptarse a nuevas modalidades, implementaciones innovadoras, uso de recursos, aplicaciones móviles, manejo de diseño instruccional y uso de dispositivos tanto portátiles como de escritorio. Por lo tanto, con esta experiencia, se necesita reflexionar, evaluar y ejecutar acciones para prepararse en el uso de las TIC no solo para enfrentar contingencias, sino para aumentar la distribución del aprendizaje, implementar procesos innovadores de aprendizaje, mejorar el uso de los

recursos digitales y estar en concordancia con la generación venidera, la cual tiene en su mayoría el dominio de esta tecnología que evoluciona constantemente.

Palabras clave: enseñanza, tecnologías de la información y la comunicación, COVID

\section{Adapting to technology in education: an emergency}

Revista RedCA octubre 2020 - enero 2021 ISSN: 2594-2824 Vol. 3 Núm. 8 


\begin{abstract}
After the emergence of the COVID contingency, the teaching of face-to-face classes was impossible and the emigration to the virtual mode presented challenges for teachers, who were not mostly prepared for this challenge. However, ICT training is required for designing and implementing innovative processes consistently. This leads us to think about the need for participants to get adapted to new modalities, innovative implementations, digital resource usage, mobile applications, instructional design management, and the use of both portable devises and desktop equipment. Hence, with this experience, we need to reflect, evaluate and execute actions to be trained for the use of ICTs not only to face contingencies, but to increase the distribution of learning, implement innovative learning processes, improve the use of digital resources and be in line with the generations, which has habitually the mastery of this constantly evolving technology.
\end{abstract}

Palabras clave: teaching, Information and communication technologies, COVID

\title{
La inesperada necesidad de las TIC.
}

El ser humano vive en un mundo globalizado y se puede beneficiar de la interacción constante con otras culturas, así como también podría perjudicarse al momento de la conexión que tenemos tan fuerte los seres humanos. Tal es el caso del surgimiento de la enfermedad COVID-19. La transmisión fue inminente y se probó la vulnerabilidad del ser humano en diferentes áreas de nuestra forma de vida. Queda demostrado con los hechos que todo se globaliza incluyendo los efectos de una pandemia, la cual afecta el arte, la ciencia, la cultura, la economía, entre otros ámbitos. Sin embargo, este fenómeno nos hizo comprender la importancia que tiene la asimilación cultural de nuevas formas de aprendizaje digital, nuevos ambientes de aprendizaje a los cuales aún gran cantidad de docentes no se acostumbra, no se adapta o se muestra renuente a incluir como parte de su práctica.

\footnotetext{
"Vivimos en un mundo globalizado que no para de cambiar. La tercera revolución industrial, la de las nuevas tecnologías, ha traído consigo cambios en ámbitos como la economía, el comercio, la publicidad, el acceso a la información y la participación ciudadana. Todos estos cambios han dado lugar a la denominada sociedad de la
}

Revista RedCA octubre 2020 - enero 2021 ISSN: 2594-2824 Vol. 3 Núm. 8 
información que se basa en los progresos tecnológicos, es decir, se confiere a las Tecnologías de la Información y la Comunicación (TIC) el poder de convertirse en los nuevos motores del desarrollo y del cambio social."(Cuenca et al., 2011, p. 14)

Esencialmente esta separación entre cada uno de los pobladores exigió al gobierno de este país a implementar sistemas de aprendizaje a distancia y virtual. Además, otros problemas han hecho que otras naciones cubrieran necesidades de capacitación con sistemas de aprendizaje innovadores. La alta densidad de población y las actividades humanas han hecho que el aprendizaje virtual sea una de las modalidades más recurridas en países desarrollados y eventualmente en los no desarrollados. "En 1910 W.A. Grundy, Inspector Mayor de Sanidad de Nueva Gales del Sur (Australia), ante las dificultades que tenía para formar a los inspectores de sanidad con destino en zonas rurales, impartió clases por correspondencia a nueve de ellos con cierto éxito"(García, 1999, pag. 22). Esto es un ejemplo de cómo la necesidad produce la sinergia para procesos innovadores para integrar la educación a distancia como un medio de distribución del aprendizaje. Posteriormente su evolución se inició con nuevos medios educativos. "En 1951 nacen las Escuelas Australianas del Aire que posibilitan que niños geográficamente aislados participen de la enseñanza diaria con un profesor y otros niños a través de la radio"(García, 1999, pag. 23).

Por otra parte algunos países no lo vieron como una necesidad, aunque hubiera una demanda prevaleciente de innovación educativa con respecto a la evolución tecnológica. A colación, vendría retomar la perspectiva de actualización ante los diferentes cambios en el uso de las tecnologías de la información y la comunicación (TIC), ya que

[e]l cambio progresivo que está experimentando el sistema económico en la actualidad es producto del crecimiento científico y tecnológico que se extiende de forma acelerada, la importancia de este tema radica en la necesidad de que las instituciones formales, como las empresas y las universidades, se den cuenta de la imperiosa necesidad del fenómeno que trae aparejada la innovación y renovación de la tecnología en diversos niveles, porque necesitan asimilar estos cambios para poder permanecer cada vez más en los mercados nacionales e internacionales altamente competitivos(Salcido et al., 2019, p. 17). 
Si no se observar la realidad de un atraso en la asimilación del uso de las tecnologías de la información como parte de la praxis docente se corre el riesgo de enfretarse a situaciones complejas de adaptación emergente ante situaciones de necesidad real, tal como lo que se vive con la contingencia ante el COVID 18. Por ende, se necesita de entes activos y no testigos de la evolución que ha permito resolver de cierta manera la problemática que ha surgido al momento de encontrarnos aislados en prevención por medio del distanciamiento social.

\section{La prevención de eventuales dificultades.}

Las autoridades deben tomar las decisiones adecuadas y seguir los estándares internacionales requeridos de la UNESCO (UNESCO-IEU, 2010) Es imperante la capacitación en el uso de las TIC, mejoramiento de la integración del equipo tecnológico, uso de la tecnología para la sustentabilidad, integración de los docentes en procesos educativos ligados a la computadora y al uso de móviles para fines educativos, el desarrollo de administración de base de datos escolares automatizados, uso de plataformas virtuales, uso de herramientas de comunicación sincrónica/asíncrona y la implementación de procesos innovadores de aprendizaje virtual.

\section{La integración tecnológica se analiza y planifica.}

Las medidas acertadas en el uso de las TIC para la sustentabilidad, para la implementación de modalidades mixtas y virtuales, la agilización de sistemas de organización e interacción escolar, el manejo de herramientas sincrónicas y asíncronas, la integración de evaluacones formativas y sumativas basadas en medios digitales, el uso de plataformas, de herramientas de aprendizaje y recursos digitales requiere análisis y planeación que pueda cubrir las demandas existentes tanto de los docentes como de los estudiantes y por ende tener un efecto de consistencia en los procesos innovadores a largo plazo. Al momento de realizar las actividades académicas dejar la esfera protectora de los procesos tradicionales en donde prepondera la cátedra por medio de recursos impresos, libro de apoyo, clases presenciales y tomar el reto de integrar las TIC. Además, estar abierto a la oportunidad de capacitarse en el uso de plataformas, uso de redes sociales, uso de procesos 
innovadores para el aprendizaje, entre otros temas relacionados por medio de cursos disponibles tanto en la red como en la institución. Es decir, "se hace necesario modificar los esquemas tradicionales de diseño y planificación de cursos y materiales para la enseñanza y utilizar las herramientas tecnológicas bajo una óptica abierta y de adaptación a los nuevos modelos de aprendizaje” (Góngora Parra \& Martínez Leyet, 2012, p. 342).

A principios del nuevo milenio, la evolución tecnológica representaba ya un aceleramiento de los sistemas de comunicación, la portabilidad y los medios masivos. Por ende, el reto de enfrentar nuevos ambientes de aprendizaje con estos medios fue dificultoso, en especial para todos aquellos no nativos digitales. Hemos evolucionado en esta virtualidad no solo en el uso de herramientas digitales, sino en el cómo las usamos y cuál es nuestra conducta con respecto a la interacción que se da en un proceso de aprendizaje virtual. Es por ello por lo que toma importancia crear una retrospectiva de la transmisión de códigos de conducta, comunicación clara, intención comunicativa que se da al momento de utilizar las herramientas de comunicación que tenemos como enfoque para facilitar los contenidos ya que representa la adaptación a nuevas etiquetas de conducta, habilitaciones tecnológicas y desarrollo crítico para la selección de recursos, herramientas y plataformas educativas digitales.

\section{Se actúa bajo la necesidad.}

Las inclemencias son las que realmente nos hace responder con mayor efectividad y este es el caso de una gran cantidad de problemas en la historia que han propiciado el desarrollo de la educación e incluso la adaptación a modalidades de aprendizaje diferentes. "[L] os países de grandes dimensiones en los que las distancias son enormes, fueron buenos bancos de prueba para el nacimiento de proyectos de enseñanza por correspondencia"(García, 1999, p. 21). Tal es el caso de Australia, país que tiene una densidad de población de tres habitantes por kilómetro cuadrado. Su geografía lo orilló a tener un sistema de educación a distancias y posteriormente virtual desde que planteó su crecimiento y desarrollo educativo. Precisamente fueron tomando como base de distribución del aprendizaje las tecnologías existentes en su momento, tales como la radio, el teléfono, la 
televisión, la computadora y los sistemas de videoconferencia. No es de extrañarse que el autor de una de las plataformas de uso libre más usada hasta ahora sea un australiano, Martin Dougiamas.

Las consideraciones sobre el uso de las TIC en nuestra época pueden tener tendencias sobre el rechazo a las mismas o la aceptación, integración y asimilación de ellas a nuestras praxis. Sin embargo, los hechos son duros y las acciones inaplazables. La evolución tecnológica siempre ha abarcado nuestras esferas sociales, económicas, políticas y sociales y la humanidad se ha adaptado a ésta.

[E]l aprendizaje electrónico es el proceso de entrega de información para la educación a través de medios electrónicos, que incluyen satélite, Intranet, Internet, CD-ROM e incluso soporte telefónico. En el entorno de e-learning, el 'e' 'e-coach', 'e-educator', 'efacilitador, 'e-educator', 'e-moderator', 'e-docente recuerda a todos los recursos humanos Profesionales involucrados en la enseñanza en línea que el valor agregado de la 'e' tecnológica ha transformado sin lugar a dudas muchos aspectos de su rol, pero ha preservado la esencia del trabajo. (Call \& Dudeney, 2004, p. 4)

Tal vez, las condiciones de la corriente tradicional se han podido mantener por un largo lapso y siguen siendo la base de la docencia; no obstante, la innovación educativa y el dominio de las TIC proporcionan a los docentes y los alumnos un mejor escenario para enfrentar las modalidades no tradicionales (mixta y virtual) ante los medios tecnológicos usados en la actualidad.[U1] "En definitiva, se trata de una educación mediada y esa mediación se ha venido realizando con una secuencia ajustada a la evolución de los medios"(García, 1999) que se integran a las modalidades de enseñanza y aprendizaje, a nuestra cultura digital y a nuestros saberes innovadores. Las modalidades educativas manifiestan la evolución que la educación tiene con respecto a nuestra forma de vida y al avance tecnológico que tenemos hoy en día. Como ejemplo, se recupera el caso de la tecnología móvil y la distribución de contenidos de aprendizaje por este medio. El aprendizaje por multiplataformas es ya una realidad y representa una oportunidad para los sistemas educativos actuales para invertir en la integración de la tecnología y, por ende, en las posibilidades de abrir nuevas modalidades de aprendizaje. Dicho reto recae sobre las 
instituciones educativas, las cuales integrarán la innovación educativa considerando el creciente uso de las nuevas tecnologías por parte de la comunidad estudiantil, pero sobre todo de las necesidades concretas de cada individuo en cuanto a tiempo, estilo de aprendizaje y necesidades laborales y económicas.

\section{Elementos para considerar la adaptación.}

Existe ya una generación de nativos digitales circundando en el nivel superior. Es decir, la generación " $Z$ ”, quienes manipulan con mayor capacidad la iconografía digital, las funciones de las interfaces, la navegación de las Apps, las redes sociales, la integración semántica de las multi-plataformas, la capacidad de distribución y manejo de la información y los diferentes tipos de dispositivos, entre otras vertientes con inclinación más interactiva como los ambientes de juegos interactivos virtuales (gaming), entre otros.

\footnotetext{
Nacidos en la era de la inmediatez, están acostumbrados a conseguir información de manera inmediata, lo que los lleva a tener distintas formas de percibir el mundo, por medio de las influencias del internet y las redes sociales. El realismo, el pragmatismo y una mentalidad menos idealista son características identificadas para quienes pertenecen a esta generación. La exposición al mundo de la inmediatez de las redes sociales facilita el aprendizaje del manejo de distintas situaciones de una forma más eficaz. (Dormoi, 2017, p. 35)
}

Dichos nativos crean una brecha que posiblemente se logre entrelazar en algún momento, cuando haya una paridad entre el dominio digital de los educadores con relación al de los aprendices.

\section{Los ambientes de aprendizaje virtual son una realidad.}

Otro aspecto que subyace dentro de nuestros entornos educativos es la integración de ambientes de aprendizaje virtual que incluso son ofrecidos por las instituciones como medida de cumplimiento a los requerimientos educativos internacionales. Tanto las herramientas, como el equipo y los medios se ofrecen por lo menos para darle al docente las capacidades 
para integrar las TIC. Además, no se mencionan en este escrito todos los servicios digitales a los cuales el docente, como cualquier otro poblador, tiene que aprender a usar para entablar las interacciones con el gobierno, el comercio, la industria y el entretenimiento. Es por ello que "la formación del profesoradoen el uso de las TIC debe realizarse en el contexto de los requisitos oficiales para la formación del profesorado, así como las necesidades de los futuros docentes cuando se enfrentan a las posibilidades técnicas que pueden encontrarse en su práctica diaria"(Krajka, 2006, p. 1).

\section{La planeación es esencial.}

La implementación de contenidos en ambientes virtuales requiere de una metodología rigurosa de la que nos provee el diseño instruccional, el cual da la pauta para crear materiales de aprendizaje con una guía educativa, con instrucciones detalladas que el aprendiente seguirá para elaborar actividades de aprendizaje con el fin de lograr un objetivo concreto que le posibilite la adquisición de conocimientos, habilidades y actitudes sobre un tema.

Desde los años 90 hasta la actualidad proliferan los modelos de enfoque contructivista para el aprendizaje y los desarrollos multimedia. El hipertexto y los hipermedios, característicos del gran universo de información que representa Internet, señalan a estos modelos como los más apropiados para el diseño de aprendizaje (Góngora y Martínez, 2012, p. 347).

En el diseño instruccional el margen de error en las instrucciones influye en el desempeño del estudiante, pues es un contacto indirecto (no presencial) y por ello no deja pie a lo implícito. Todo lo que se va a realizar por los estudiantes debe estar explícito y preciso para lograr el objetivo de aprendizaje y esto se obtiene mediante una instrucción efectiva por medio de capacitacion de

los expertos en contenido, pedagogos y docentes-asesores en diseño instruccional y uso de nuevas tecnologías, puesto que quien no tenga conocimientos de cómo planear y diseñar un programa educativo a distancia y no haya utilizado las tecnologías de la comunicación, ya sea para aprender o para enseñar, no podrá imaginar la interacción entre los estudiantes y el asesor de otra manera que no sea en la modalidad presencial (Ortiz Cáceres, 1969, p. 113).

Revista RedCA octubre 2020 - enero 2021 ISSN: 2594-2824 Vol. 3 Núm. 8 
En nuestra planeación, pensamos previamente qué contenidos se van a abordad, qué actividades van a realizar los alumnos con esos contenidos, qué actividades haré yo como docente, quiénes estarán involucrados y en qué momento, cuánto tiempo, qué harán los estudiantes entre ellos, qué harán conmigo, qué harán con los contenidos. En fin, es una serie de sucesos que engloba precisamente el aspecto integrador y socializador del aprendizaje y la enseñanza. Incluye además la concepción principal de un aprendizaje colaborativo que se va a dar con los pares y con la figura del instructor como una guía que proporciona los elementos adecuados para que el ambiente de aprendizaje sea idóneo.

Los diseños instruccionales aplicados a actividades de aprendizaje en ambientes virtuales no dan pie a la duda o el desconcierto, pues una indicación errónea propicia malas direcciones en el aprendizaje y por ende un rendimiento menor en el desempeño de los participantes.

\section{La sustentabilidad de los recursos.}

Otro factor que se ve beneficiado de la implementación tecnológica es la sustentabilidad de recursos tanto humanos como materiales. El uso de plataformas educativas, por ejemplo,

\footnotetext{
"no sólo involucra una oportunidad para agilizar los procesos de aprendizaje, sino un medio de eficientar los procesos para los cuales el docente dedica tanto tiempo y esfuerzo. Aunado a ello, se incluye también el ahorro de papel para realizar exámenes que pueden llevar a la institución a afectar el ambiente en que vivimos mediante el uso irracional de papel. De esta manera se considera que hay tres categorías en las cuales la implementación de plataformas puede beneficiar a la práctica docente: a) recursos humanos, b) tiempo y c) sostenibilidad (Castillo et al., 2016, p. 2).
}

Un humano puede verse beneficiado de ahorro de tiempo y esfuerzo al momento de responsabilizar a la computadora de procesos mecanicistas, organizativos y de índole repetitivo; Aunque hay procesos imposibles para la máquina, por ejemplo, la computadora no ejecuta procesos subjetivos que necesitan la sensibilidad y apreciación del ser humano. 
Para agregar, el ahorro de los recursos materiales, tales como papel y almacenamiento de espacio es otra de las ventajas del uso de la tecnología.

\section{La creciente accesibilidad a herramientas de autoría para la educación.}

Aunque la literacidad digital es primordial para el docente, el avance de la ciencia computacional y de accesorios portátiles ha permitido que haya disponible mayor número de herramientas de autoría para el desarrollo de ejercicios interactivos, manejo de datos, elaboración de exámenes cortos, edición de audio y video, elaboración de mapas mentales, juegos interactivos educativos, entre otros, por lo tanto "[c]on las herramientas de autor el creador de una aplicación se preocupa fundamentalmente del diseño, de su aspecto y casi nada de la programación” (Sánchez, 2003, p. 89). Este avance orientado a una interfaz más amable para los diseñadores instruccionales ha permitido que los impedimentos técnicos dejen de existir gradualmente. Es decir, conforme avanza el tiempo, un docente es cada vez más capaz de desarrollar materiales de aprendizaje basados en computadora, en la Web o en tecnología móvil.

\section{La cultura del aprendizaje para toda la vida.}

Otro de los aspectos que integraron a la tecnología de la información y la comunicación en la educación es el objetivo de tener un repertorio de posibilidades para que existiera educación continua, capacitación para profesionistas, oferta educativa de licenciaturas y posgrados para los trabajadores, cursos de capacitación, entre otros. Este fenómeno también hizo crecer el número de individuos dedicados a integrar las TIC a la educación. Asimismo, se combinó con la necesidad del estado de masificar la educación, de tener mayores modalidades de aprendizaje para llegar a más usuarios que se encuentran con la necesidad de actualizarse o de estudiar una profesión.

\section{Conclusión}

Mundo globalizado requiere docentes preparados en TIC. Sin lugar a dudas, al observar el panorama de la evolución de nuestra especie, aunado a la forma en la cual 
interactuamos y a las necesidades globales, la capacitación en el uso de las TIC es una necesidad imperante para enfrentar situaciones no solo de emergencia, sino para enriquecer nuestro sistema educativo con modalidades más apropiadas para las nuevas generaciones y para los diferentes tipos de alumnos. Es por ello que se necesita diversificar las modalidades de aprendizaje y aumentar nuestro dominio digital. "el uso de la tecnología debe ser guiado y dosificado por expertos, ya que en la actualidad hay una suma innumerable de información y diversos recursos y/o aplicaciones innovadoras.

Estos pueden contribuir a la formación académica de los estudiantes, desde cualquier nivel de estudios. Sin embargo, el control adecuado del uso de estos procesos innovadores de aprendizaje debe preponderar al momento de su implementación, ya que los usuarios pueden estar desorientados al momento de aplicarlos sin una asimilación adecuada de sus potencialidades(Guevara, Rodríguez y Salazar, 2019, pp. 9-10). Las instituciones de educación superior actualmente deben contar con diferentes modalidades de aprendizaje con el fin de dar oportunidad a la comunidad estudiantil de superarse académicamente. Tenemos una gran diversidad de estilos de aprendizaje e inteligencias múltiples que presentan la oportunidad de aprender y desarrollar inteligencias y potencialidades de acuerdo con el ambiente que les proporcionamos.(Gardner, 1987). Esto nos lleva a considerar situaciones individuales que necesitan atención y sobre todo una reestructuración en los sistemas educativos actuales.

Podemos encontrar hoy modalidades que han tomado diferentes formas de distribución del aprendizaje y han variado los espacios físicos con los virtuales. También se han creado instituciones íntegramente virtuales que ofrecen educación formal e informal a través de Internet. Otros sistemas presenciales combinan las herramientas que suministra el internet para usar la tecnología y enriquecer su práctica en la enseñanza. También es necesario tener una diversidad de ambientes de aprendizaje tanto sincrónico como asíncrono que permita la interacción socioafectiva en el marco de un enfoque comunicativo y humanista. Además, los recursos que se ofrecen a los alumnos se necesitan refinar técnicamente, en cuestión de amigabilidad del usuario, aumento del ancho de banda, capacitación más orientada al uso de las TIC para los docentes.

Revista RedCA octubre 2020 - enero 2021 ISSN: 2594-2824 Vol. 3 Núm. 8 
Es decir, adaptarse a los cambios que has surgido y seguirá habiendo. Por lo cual, el docente necesita saber evaluar las herramientas de autoría para la mejor toma de decisiones al momento de seleccionar las herramientas digitales para su implementación educativa innovadora y, por ende, evaluar constantemente las herramientas digitales que se encuentran disponibles en la red en función de las capacidades técnicas de la institución, el docente, los alumnos y de las capacidades académicas que ofrece. En nuestros días las exigencias para enfrentar las necesidades profesionales son más demandantes. Sobre todo, en el aspecto de la interdependencia, el enfoque ecológico que ve como sistema integral e interrelacionado el mundo en que vivimos y en el cual interactuamos no sólo uno con otros, sino la naturaleza, los recursos, la flora, la fauna y los sistemas humanos en relación con las artes, las ciencias y la tecnología.

\section{Referencias bibliográficas}

Call, B., y Dudeney, G. (2004). E-Educator. Octubre, 2004. https://Itsig.iatefl.org/wpcontent/uploads/cr0507.pdf

Castillo, F., Rivera, M., Hernández, M., Martínez, J., \& Guevara, I. (2016). Moodle como medio para eficientar la evaluación y la sostenibilidad. Revista Iberoamericana de Producción Académica y Gestión Educativa PAG, 04, 25. https://www.pag.org.mx/index.php/PAG/article/view/454/494

Cuenca, A., Herrera, O., \& Haro, P. De. (2011). ¿Que necesito aprender para ser Teleformador? 1, 177.

Dormoi, A. (2017). El poder de la comunicación de los Centennials. Universidad de Palermo.

García, L. (1999). Historia de la Educación a Distancia (History of Distance Education). Revista Iberoamericana de Educación a Distancia, 2(1), 8-27. http://revistas.uned.es/index.php/ried/article/view/2084

Gardner, H. (1987). The theory of multiple intelligences. Annals of Dyslexia, 37(1), 19-35. 
https://doi.org/10.1007/BF02648057

Góngora, Y., \& Martínez L. O. (2012). Del diseño instruccional al diseño de aprendizaje con aplicación de las tecnologías. Education in the Knowledge Society (EKS), 13(3), 342-360. https://doi.org/10.14201/eks.9144

Guevara, Isaí. Rodríguez, Alejandro. Salazar, E. (2019). Educación Innovación tecnológica y Auto-aprendizaje (1st ed.). Brujas.

Krajka, Jarosław. (2006). Jarosław Krajka ICT in the Foreign Philology Curriculum Towards a Systematic Approach to E-Teacher Training.

Ortiz, Cáceres. (1969). Gestión curricular en las escuelas con tecnologías de la información y la comunicación. Sistematización de algunas experiencias en Chile. Perfiles Educativos, 35(141). https://doi.org/10.22201/iisue.24486167e.2013.141.40529

Salcido, R. E., Guadalupe, L., Dise, M., Toda, N., Editorial, A., \& No, H. (2019). Innovación tecnológica en las prácticas educativas (1a ed.). Amapsi.

Sánchez, et al. (2003). Aplicaciones educativas de las tecnologías de la información y la comunicación. comunicación. Centro de Publicaciones. Ministerio de Educación y Ciencia.

UNESCO-IEU. (2010). Medición de las tecnologías de la información y la comunicación (TIC) en educación - manual del usuario. http://www.uis.unesco.org 